\title{
OPERACIONALIZAÇÃO DA LOGÍSTICA REVERSA EM SOLUÇÕES CIRCULARES: MAPEAMENTO DAS RESPONSABILIDADES DOS STAKEHOLDERS
}

Adriana Hofmann Trevisan (adriana.engamb@outlook.com) - Departamento de Engenharia de Produção, Escola de Engenharia de São Carlos, Universidade de São Paulo.

Daniel Guzzo da Costa (guzzoo@gmail.com) - Departamento de Engenharia de Produção, Escola de Engenharia de São Carlos, Universidade de São Paulo.

Janaina Mascarenhas Hornos da Costa (janainamhcosta@gmail.com) - Departamento de Engenharia de Produção, Escola de Engenharia de São Carlos, Universidade de São Paulo.

\section{RESUMO}

A economia circular propõe uma mudança no atual sistema linear de produção e consumo, para uma economia que busca a circularidade de materiais continuamente. Assim, para viabilizar um modelo de negócio pautado na economia circular, um sistema de logística reversa é indispensável. Porém, as atividades relacionadas a logística reversa implicam relações complexas entre inúmeros stakeholders. Nesse sentido, o objetivo dessa pesquisa é mapear os stakeholders diretamente envolvidos em um sistema de logística reversa de um equipamento eletromédico, buscando entender as suas responsabilidades na operacionalização desse sistema. Para isso, é realizado um estudo de caso em uma empresa que manufatura e comercializa equipamentos eletromédicos, localizada na cidade de São Carlos-SP. Os resultados da pesquisa indicaram múltiplas responsabilidade dos stakeholders para a operacionalização dos serviços que envolvem manutenção, remanufatura e reprocessamento de um produto modular. Os mapas apresentados podem ser detalhados envolvendo outros membros da cadeia de valor ou ser desenvolvidos para outros tipos de produtos com serviços associados.

Palavras-chave: Logística Reversa; Sistema Produto-Serviço; Economia Circular

Área: Economia circular e o desenvolvimento de produtos e serviços

\section{INTRODUÇÃO}

O modelo linear de produção e consumo, sustentado pelas atividades de "extrair, transformar e descartar", foi imprescindível para a evolução da economia global. Porém, qualquer sistema que baseia-se no consumismo, e não no uso sustentável e restaurativo dos recursos, prevê consideráveis perdas ao longo da cadeia de valor (AZEVEDO, 2015). Em vista disso, a economia circular propõe uma ruptura do sistema linear para um modelo que diminua o consumo de energia e matérias-primas e, ao mesmo tempo, maximiza a utilização de recursos renováveis ou recicláveis (AZEVEDO, 2015; ELLEN MACARTHUR FOUNDATION, 2015).

Soluções no contexto da Economia Circular buscam fechar os ciclos de materiais, através da utilização dos recursos repetidamente (YUYAN, 2010), de uma forma que aumente a entrega de valor ao cliente e a geração de lucros para as organizações (ELLEN MACARTHUR FOUNDATION, 2015; fig.1). Dessa forma, os ciclos técnicos, composto por componentes e produtos manufaturados, precisam estar em circularidade, por meio do reuso dos produtos, de módulos ou componentes dos produtos e materiais (VAN DEN BERG; BAKKER, 2015). 
Todavia, diversos autores argumentam que para viabilizar um modelo de negócio pautado na economia circular, um suporte de Logística Reversa (LR) necessita ser estabelecido pela cadeia de valor (YUYAN, 2010; ELLEN MACARTHUR FOUNDATION, 2015; LEWANDOWSKI, 2016), pois é através da LR que o produto, ou seus componentes, podem ser reparados, remanufaturados, reciclados ou eliminados, de acordo com o gerenciamento do produto no fim de vida (GOVINDAN; SOLEIMANI; KANNAN, 2015).

Porém, as atividades relacionadas a LR implicam relações complexas entre múltiplos stakeholders e a empresa fabricante do produto (ÁLVAREZ-GIL et al., 2007). Logo, verificar o papel dos stakeholders dentro desse contexto é uma forma de entender as suas responsabilidades e favorecer a definição e operacionalização da logística reversa no contexto de um modelo econômico circular.

Nesse sentido, este trabalho tem como objetivo identificar os stakeholders diretamente envolvidos em um sistema de logística reversa de um equipamento eletromédico, a fim de demonstrar as interações e interdependência entre eles. $O$ setor de equipamentos eletromédicos foi escolhido para essa pesquisa, por ser uma área promissora e propulsora de desenvolvimento tecnológico (MORELI et al., 2010), com consideráveis possibilidades de soluções circulares, e por possuir um mercado com grandes expectativas de crescimento entre produtos remanufaturados e recondicionados (IRRC INSTITUTE, 2015; PARKER et al., 2015). Como, por exemplo, as iniciativas de equipamentos médicos recondicionados pela Philips (2014, p. 13), Johnson \& Johnson (IRRC INSTITUTE, 2015) e Toshiba (IRRC INSTITUTE, 2015).

\section{REVISÃO DA LITERATURA}

Dentre as diversas conceituações do termo logística reversa, a que se apresenta mais consolidada na literatura é a definição dos autores Rogers e Tibben-Lembke de 1998. De acordo com eles, a LR é caracterizada como "o processo de planejamento, implementação e controle da eficiência e custo efetivo do fluxo de matérias-primas, estoques em processo, produtos acabados e as informações correspondentes do ponto de consumo ao ponto de origem, com a finalidade de recapturar ou criar valor ou eliminação adequada".

A partir dessa definição, é possível pressupor a existência de inúmeros serviços e atividades associados a LR, para que ocorra a criação de valor, maximização do ciclo de vida ou disposição adequada de materiais. Fleischmann et al. (2000) apontam etapas específicas e recorrentes em redes de recuperação de produtos, para que eles possam ser utilizados novamente. Essas etapas consistem em:

- Coleta: Atividades de recolha do produto físico do mercado. Em geral, incluindo atividades como aquisição, transporte e armazenamento.

- Inspeção/Separação: Operações que determinam se um produto pode, de fato, ser reutilizável (ou eliminável) e de que maneira.

- Reprocessamento: Consiste na etapa de transformação do produto utilizado e um produto capaz de ser utilizável novamente.

- Disposição: Destinado a produtos que não podem ser recuperados por motivos técnicos ou econômicos.

- Redistribuição: Atividades que direcionam o produto, já recuperado, para potenciais mercados que o disponibilizarão para novos usuários.

Raci e Shankar (2005) argumentam que durante a fase de desenvolvimento de produto, o conteúdo do material e a estrutura do produto precisam ser considerados, pois o tipo de 
material e a tecnologia empregada na fabricação, determinam a extensão da recuperação após o fim de uso/vida do produto. Dessa forma, para que um bem permaneça utilizável por mais tempo, é necessário levar em consideração todo o seu ciclo de vida.

Jun et al. (2007) apresentam três fases principais relacionadas ao ciclo de vida do produto. Essas fases são: Beginning of life (BOL), fase de início de vida que inclui design e produção; Middle of life (MOL), relacionadas as etapas de logística (distribuição), uso, serviços e manutenção; e o End of Life (EOL), que inclui logística reversa, remanufatura, reutilização, reciclagem e eliminação.

Dentre as atividades do MOL, os serviços de manutenção podem ser divididos em três tipos básicos, de acordo com a autora Swanson (2001), que são: Manutenção Reparativa, que ocorre após o produto apresentar alguma falha; Manutenção Proativa, dividida entre preditiva e preventiva, que está associada a evitar quebras de equipamentos; e a Manutenção Agressiva, que é uma abordagem que busca melhorar o desempenho do equipamento e, ao mesmo tempo, evitar possíveis falhas.

Os serviços de manutenção são importantes para diversas operações, pois eles estendem a vida do equipamento, melhoram a disponibilidade do equipamento e o mantém em condições adequadas de uso (SWANSON, 2001). Da mesma forma, as atividades do EOL são fundamentais para a recuperação de ativos e possibilitam que o produto seja utilizado além de um único ciclo, por meio da LR e demais processos pertinentes a fase de fim de vida.

Deste modo, para a identificação dos stakeholders e suas responsabilidades em um sistema de logística reversa, serão consideradas as atividades relacionadas aos serviços de manutenção, reparação, remanufatura e descarte do produto e seus componentes. É imperativo manter em consideração na pesquisa que as atividades de gerenciamento de serviços, como o reparo, dependem fortemente de uma logística reversa eficaz (AMINI et al., 2005).

\section{METODOLOGIA}

Para o desenvolvimento dessa pesquisa, inicialmente, houve uma revisão da literatura, para compreensão de conceitos-chave relacionados a temática de logística reversa, economia circular e gestão do ciclo de vida do produto, incluindo BOL, MOL e EOL. A revisão da literatura contribuiu para dar suporte teórico a pesquisa.

Posteriormente, um protocolo de investigação foi desenvolvido e aplicado em um caso prático de logística reversa de um equipamento eletromédico. O protocolo foi estruturado de acordo com as etapas de recuperação de produtos, apresentadas por Fleischmann et al. (2000), e segundo os serviços de manutenção, presentes no trabalho de Swanson (2001), sobre estratégias de manutenção de equipamentos.

O estudo de caso foi escolhido como método para essa pesquisa por ser uma estratégia que se concentra na compreensão da dinâmica de um determinado local (EISENHARDT, 1989) ou situação. E por possibilitar que os pesquisadores estivessem expostos com os problemas reais acerca do tema. Assim, uma entrevista guiada de maneira semiestruturada propiciou que os pontos importantes fossem trazidos para discussão, de acordo com as interações pesquisadores - entrevistado.

O protocolo, acima descrito, foi totalmente esgotado, a partir da entrevista que ocorreu com o coordenador de serviços e pós-vendas de uma organização que fabrica e comercializa dispositivos médicos, localizada na cidade de São Carlos - SP. A organização será denominada, nessa pesquisa, como Empresa A. 
Após isso, houve discussões e reflexões entre os pesquisadores, de modo a caracterizar as interações e interdependência entre os diferentes stakeholders, no processo de logística reversa do produto em questão. Assim, foram identificados os stakeholders diretamente envolvidos nos serviços pertinentes ao período de uso do equipamento pelo cliente (Middle of life), bem como, as atividades relacionadas as soluções de fim de vida do produto e seus componentes (End of Life).

Dessa maneira, todo fluxo reverso de materiais, no sentido de operacionalizar os ciclos técnicos, foram considerados como parte da logística reversa. E todos os serviços que visavam garantir a funcionalidade do produto, pelo maior tempo possível, também foram pontuados nesse estudo.

Para finalizar esse processo, foi elaborado um mapa dos stakeholders, baseado nas orientações dos autores Stickdorn e Schneider (2011), e apresentado ao entrevistado, que contribuiu para o alcance dos resultados e que validou as informações anteriormente coletadas.

\section{RESULTADOS}

A partir de entrevista com o coordenador de serviços e pós-vendas da Empresa A, e de iterações de discussões entre os pesquisadores, mapeamento dos stakeholders e validação dos pesquisadores com o entrevistado, apresentamos o seguinte resultado.

\subsection{Estudo de caso e stakeholders}

A Empresa A possui aproximadamente 100 funcionários e as áreas industrial e de administração estão localizadas em um mesmo local. No mercado nacional, a Empresa A estabelece uma relação Business to Business com seus clientes, na qual vende os produtos para empresas prestadoras de serviço. Além da venda do produto, ocorre o oferecimento de garantia, serviços adicionais de manutenção preditiva, manutenção reparativa e reposição de peças. A oferta de valor identificada caracteriza um Sistema Produto Serviço do tipo orientado ao produto, de acordo com a classificação de Tukker (2004).

O equipamento médico que é foco de estudo neste trabalho consiste em uma plataforma e módulos com diferentes funções de aplicação nas áreas de dermatologia, cirurgia plástica e vascular. Por caracterizar um produto modular, a plataforma e os módulos possuem diferentes ciclos de vidas e podem ser tratados separadamente. A empresa desenvolve, manufatura, monta e fornece peças sobressalentes para o produto em questão. É também importante ressaltar a existência de duas versões da plataforma (V1 e V2) e de que os módulos projetados para a plataforma nova não são aplicáveis para a plataforma antiga.

Além da Empresa A, foram mapeados quatro organizações e três papéis centrais para a realização dos serviços oferecidos. O mapa de stakeholders está apresentado na Figura 1, onde as organizações estão representadas nos blocos com fundo escuro e os papéis estão com o fundo claro. Os traços representam a conexão entre as organizações e papéis para a criação de valor por meio dos serviços. A Empresa de logística é o único stakeholder identificado que tem relação com o cliente sem intermédio da Empresa A, por ser quem faz a movimentação do produto. O papel de assistência técnica é central na empresa para a realização dos serviços identificados, fazendo a interface com os clientes e pessoal de linha de produção para serviços de reparo mais elaborados. 
Figura 1. Mapa de stakeholders envolvidos na logística reversa do produto. Fonte: Elaboração própria (2017).

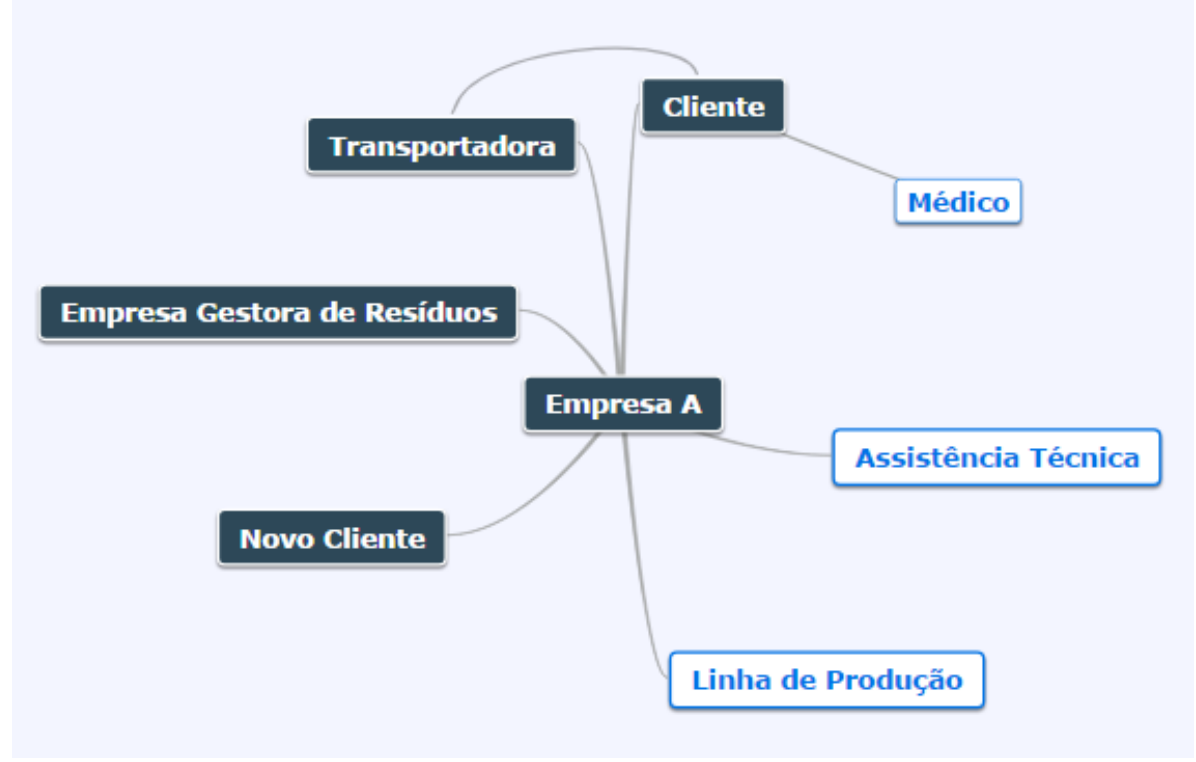

\subsection{Serviços prestados pela empresa A que envolvem Logística Reversa}

Foram identificados sete serviços que envolvem atividades de logística reversa. Os serviços estão apresentados na Figura 2. Nesta figura, os serviços estão organizados de acordo com as atividades para manter o funcionamento pelo maior tempo possível do produto (MOL) e em soluções de fim de vida (EOL). Cada stakeholder está representado por uma cor. A descrição das responsabilidades estão disponíveis na Tabela 1.

Os quatro primeiros serviços representados são referentes à plataforma e os três últimos ao módulo. Serviços semelhantes referentes à plataforma e aos módulos foram representados separadamente pelo fato de possuírem tamanho diferentes, o que influencia bastante o transporte. Por exemplo, apesar de os serviços de manutenção preventiva da plataforma e dos módulos possuírem o mesmo gatilho para serem iniciados (acontecem anualmente), para a calibração da plataforma é necessário que um membro da assistência técnica vá ao cliente, enquanto que o módulo pode ser enviado para a empresa.

Deste modo, apresentamos os sete serviços identificados e as peculiaridades de cada um:

1. Serviço de manutenção preventiva - plataforma: A calibração manual da plataforma, que acontece anualmente, é o ponto inicial para a manutenção preventiva. Alguns componentes são tratados como consumíveis da plataforma. Estes devem ser trocados de acordo com a vida útil do componente. Há a possibilidade do cliente levar a plataforma até a Empresa A, e isto acontece para clientes da região.

2. Serviço de manutenção reparativa - plataforma: O serviço é iniciado quando o usuário (médico) identifica que a plataforma parou de funcionar. Enquanto sob garantia, a manutenção da plataforma respeita o acordo de nível de serviço estipulado em contrato, caso contrário é necessário contratar o serviço. O custo de deslocamento do técnico é considerado.

3. Serviço de descarte - plataforma: O serviço é iniciado quando é identificado que os custos de reparo são proibitivos para o cliente e o produto já não está sob garantia. Apesar da quantidade de peças descartadas ser bem maior, busca-se remanufaturar o máximo de peças e consumíveis possível na plataforma. Até o momento alguns poucos casos ocorreram, referiam-se à plataforma V1 (antiga) e em todos eles o cliente levou a plataforma á empresa. 
Figura 2. Mapa de responsabilidades dos Stakeholders dos serviços adicionais ao produto. Fonte: Elaboração própria (2017).

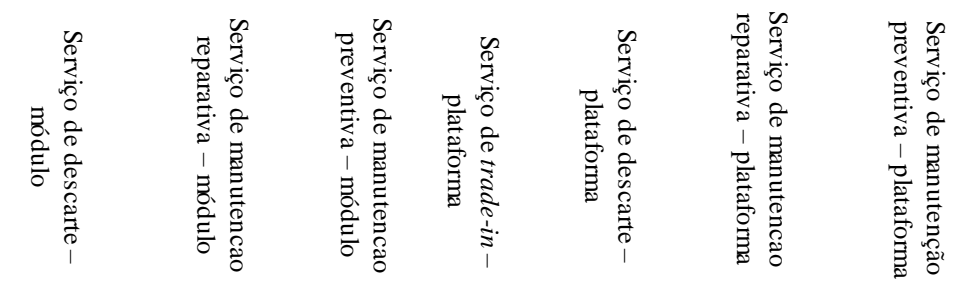

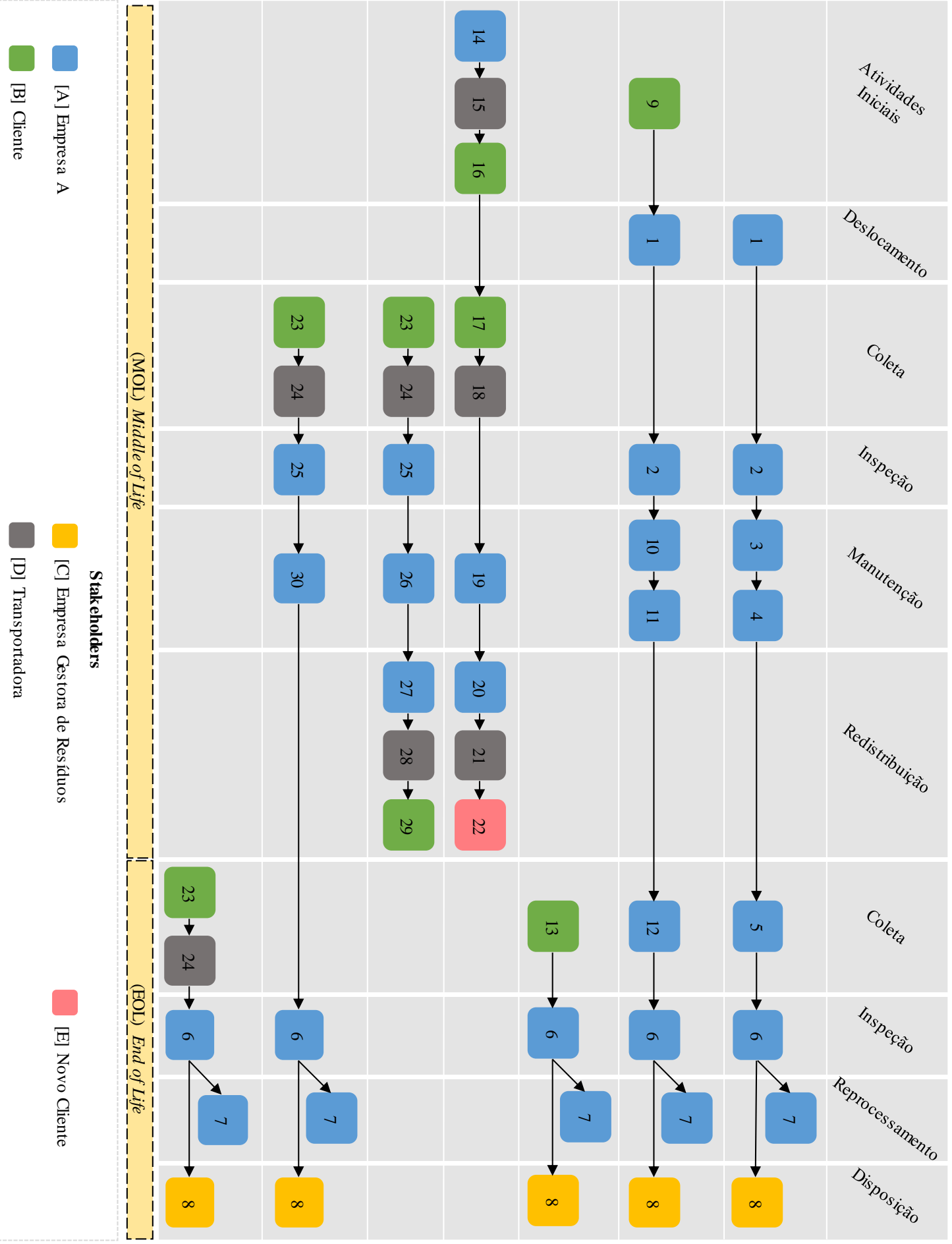


4. Serviço de Trade-in - plataforma: O serviço é iniciado quando o cliente possui a plataforma antiga e tem interesse em adquirir uma plataforma nova. Neste serviço, a plataforma antiga é remanufaturada e realocada para um novo cliente. Nos casos existentes, tal cliente é um hospital que funciona como instituição de ensino e que adquire a plataforma remanufaturada a preços abaixo do mercado. Há a possibilidade de o produto não poder ser remanufaturado e redistribuído por estar obsoleto.

5. Serviço de manutenção preventiva - módulo: O serviço acompanha a frequência anual da calibração do módulo.

6. Serviço de manutenção reparativa - módulo: O serviço é iniciado quando o usuário (médico) identifica que o módulo parou de funcionar. Regras de garantia e nível de serviço também se aplicam ao módulo. O módulo é enviado para a empresa e, por possuir componentes de alto grau de complexidade de manufatura, os serviços são feitos in house. Há a possibilidade de aluguel temporário de outro módulo no caso de reparo.

7. Serviço de descarte - módulo: Havendo custo proibitivo para reparo, inicia-se o processo de descarte. O serviço envolve o envio do módulo via transportadora.

Nota-se que a vigência da garantia e o nível de serviço acordado são pontos importantes para definir as responsabilidades de cada stakeholder em cada caso. Além disso, a distância entre o cliente e a Empresa A influencia notavelmente.

A plataforma é movimentada o mínimo possível, pois é a parte do produto de maior valor agregado e, portanto, o risco do transporte é mais alto. Quando é necessário inspecioná-la, o pessoal da assistência técnica da Empresa A que vai até o cliente. Existe a ocorrência de clientes que levam a plataforma até empresa para serviços e descarte devido ao custo envolvido no transporte de um bem de alto valor agregado.

Tabela 1. Descrição das responsabilidades dos stakeholders.

\begin{tabular}{|l|l|l|}
\hline \multicolumn{2}{|l|}{ RESPONSABILIDADES } & Stakeholder responsável \\
\hline$\#$ & Descrição & Empresa A \\
\hline 1 & Deslocar assistência técnica ao Stakeholder [B] & Empresa A \\
\hline 2 & Inspecionar plataforma in loco & Empresa A \\
\hline 3 & Calibrar plataforma in loco & Empresa A \\
\hline 4 & Trocar consumíveis (de acordo com a vida útil) in loco & Empresa A \\
\hline 5 & Retornar consumíveis ao Stakeholder [A] & Empresa A \\
\hline 6 & Inspecionar e separar peças e consumíveis & Empresa A \\
\hline 7 & Remanufaturar peças / consumíveis & Empresa Gestora de Resíduos \\
\hline 8 & Descartar peças / consumíveis & Cliente \\
\hline 9 & Chamar para verificar problema & Empresa A \\
\hline 10 & Realizar manutenção da plataforma in loco & Empresa A \\
\hline 11 & Substituir peças defeituosas & Empresa A \\
\hline 12 & Retornar peças ao Stakeholder [A] & Cliente \\
\hline 13 & Levar a plataforma ao Stakeholder [A] & Empresa A \\
\hline 14 & Solicitar ao Stakeholder [D] transporte da plataforma v2 & Transportadora \\
\hline 15 & Enviar plataforma V2 ao Stakeholder [B] & Cliente \\
\hline 16 & Receber plataforma V2 & Cliente \\
\hline 17 & Solicitar ao Stakeholder [D] transporte da plataforma v1 & Transportadora \\
\hline 18 & Enviar plataforma V1 ao Stakeholder [A] & \\
\hline
\end{tabular}




\begin{tabular}{|l|l|l|}
\hline 19 & Remanufaturar plataforma V1 & Empresa A \\
\hline 20 & Solicitar transporte da plataforma ao Stakeholder [E] & Empresa A \\
\hline 21 & Enviar plataformaV1 remanufaturada ao Stakeholder [E] & Transportadora \\
\hline 22 & Receber plataforma V1 remanufaturada & Novo Cliente \\
\hline 23 & Solicitar transporte do módulo ao Stakeholder [A] & Cliente \\
\hline 24 & Enviar módulo ao Stakeholder [A] & Transportadora \\
\hline 25 & Inspecionar módulo & Empresa A \\
\hline 26 & Calibrar módulo & Empresa A \\
\hline 27 & Solicitar transporte do módulo ao Stakeholder [B] & Empresa A \\
\hline 28 & Enviar módulo ao Stakeholder [B] & Transportadora \\
\hline 29 & Receber módulo Calibrado & Cliente \\
\hline 30 & Realizar reparo no módulo e remover peças defeituosas & Empresa A \\
\hline
\end{tabular}

\section{DISCUSSÕES}

Um ponto a ser identificado no mapa apresentado é que existem duas etapas para caminhar para o fim de vida de um produto. Por um lado, existe o fim de vida da utilidade de um produto, sistema ou componente para o usuário. Quando isto acontece, atividades podem ser realizadas para retornar o produto ou parte dele à condição de uso e envolve também a logística reversa deste para retorná-lo ao middle of life (MOL). Por outro lado, no fim de vida da utilidade do produto para a empresa, a logística reversa deve ativar o outro lado da cadeia para as atividades de end of life (EOL). Neste caso, pode-se envolver parceiros de gestão de resíduos para remanufatura em outras cadeias de valor ou para reciclagem.

Assim, é interessante pontuar que ao abordar o tema logística reversa, é preciso levar em consideração tanto o fim de vida do produto, bem como o fim de uso do equipamento pelo usuário, pois um produto pode retornar ao fabricante, não necessariamente, porque chegou ao fim de vida, e sim, devido ao fato do usuário querer uma versão atualizada do equipamento, como ocorre no caso do serviço de trade-in.

Deste modo, é importante ressaltar que as atividades para a segunda, terceira e subsequentes vidas de um produto, ou componente, precisam ser também consideradas para operacionalizar a logística reversa e criar oportunidades para outros ciclos de economia circular. Para iniciativas ainda mais circulares, como o oferecimento de sistemas produto-serviço orientados ao uso ou à performance (ver Tukker, 2004), outras responsabilidades precisam ser definidas e operacionalizadas. Desta forma, o mapa de stakeholders e de responsabilidades podem tornar-se ainda mais complexos, de acordo com o tipo de produto a ser retornado. Os serviços indicam que novas organizações, papéis e responsabilidades precisam ser definidas para que a logística reversa possa operacionalizar modelos de negócio mais circulares.

\section{CONSIDERAÇÕES FINAIS}

Em um modelo econômico circular, cada ator que compõe o sistema desempenha uma função essencial para a operacionalização da logística reversa. Através desse estudo, foi possível identificar o papel de cada stakeholder e a sua interdependência na criação de valor, a partir de produtos e componentes usados e coletados, para a utilização além de um único ciclo de vida.

Além disso, notou-se, por meio do mapa, a existência de uma responsabilidade conjunta por todos os membros da logística reversa. Pôr em prática essa responsabilidade, contribui na 
garantia de que o produto seja coletado, recuperado e redistribuído. Em outras palavras, possibilita a circularidade do produto de forma eficiente.

Por se tratar de apenas um caso é importante ressaltar que existem possibilidades futuras de desenvolvimento, como o aprofundamento nas outras organizações da cadeia de valor do produto ou em mais produtos e soluções em um ramo específico como o de eletromédico.

\section{REFERÊNCIAS}

ÁLVAREZ-GIL, M. J. et al. Reverse logistics, stakeholders' influence, organizational slack, and managers' posture. Journal of Business Research, v. 60, n. 5, p. 463-473, 2007.

AMINI, M. M.; RETZLAFF-ROBERTS, D.; BIENSTOCK, C. C. Designing a reverse logistics operation for short cycle time repair services. International Journal of Production Economics, v. 96, n. 3, p. 367-380, 2005.

AZEVEDO, J. L. DE. A Economia Circular Aplicada No Brasil : Uma Análise a Partir Dos Instrumentos Legais. Xi Congresso Nacional De Excelência Em Gestão, 2015.

EISENHARDT, K. M. Building Theories from Case Study Research Published by : Academy of Management Stable URL: http://www.jstor.org/stable/258557 Linked references are available on JSTOR for this article : Building Theories from Case Study Research. v. 14, n. 4, p. 532-550, 1989.

FLEISCHMANN, M. et al. A characterisation of logistics networks for product recovery. Omega, v. 28, n. 6, p. 653-666, 2000.

FOUNDATION, E. M. Rumo à Economia Circular: O Racional de Negócio para Acelerar a Transição. p. 22, 2015.

GOVINDAN, K.; SOLEIMANI, H.; KANNAN, D. Invited Review: Reverse logistics and closed-loop supply chain: A comprehensive review to explore the future. European Journal of Operational Research, v. 240, n. 3, p. 603-626, 2015.

IRRC INSTITUTE. Driving Revenue Growth Trough Sustainable Products and Services. The Conference Board. Trusted insights for business worldwide, 2015.

JUN, H. B.; KIRITSIS, D.; XIROUCHAKIS, P. Research issues on closed-loop PLM. Computers in Industry, v. 58, n. 8-9, p. 855-868, 2007.

LEWANDOWSKI, M. Designing the business models for circular economy-towards the conceptual framework. Sustainability (Switzerland), v. 8, n. 1, p. 1-28, 2016.

MORELI, É. C. et al. Cenários Internacional e Nacional do Setor de Equipamentos Médicos , Hospitalares e Odontológicos. Relatório Técnico da fase de Diagnóstico, componente do projeto Programa de Desenvolvimento do Arranjo Produtivo Local Médico, Hospitalar e Odontológico do Município de Ribeirão Preto e Região., p. 1-36, 2010.

PARKER, D. et al. Remanufacturing Market Study. n. 645984, 2015.

PHILIPS. Rethinking the future: Our transition towards a circular economy, 2014.

RACI, V.; SHANKAR, R. Analysis of interactions among the barriers of reverse logistics. Technological Forecasting and Social Change, v. 72, n. 8, p. 1011-1029, 2005.

ROGERS, D. .; TIBBEN-LEMBKE, R. S. Going Backwards : Reverse Logistics Trends and Practices. University of Nevada. Center for Logistics Management, 1998.

STICKDORN, M.; SCHNEIDER, J. This is Service Design Thinking, 2011. 
SWANSON, L. Linking maintenance strategies to performance. Int. J. Production Economics, v. 70, p. 237-244, 2001.

TUKKER, A. Eight Types of Product Service Systems. Business Strategy and the Environment, v. 13, p. 246-260, 2004.

VAN DEN BERG, M. R.; BAKKER, C. A. A product design framework for a circular economy. PLATE conference, n. June, p. 365-379, 2015.

YUYAN, S. H. Research on the circular economy development mode of automobile replacement parts industry based on reverse logistics. 2010 International Conference on Intelligent Computation Technology and Automation, ICICTA 2010, v. 3, p. 140-143, 2010. 